\title{
Multi-Controller Design under Uncontrolled and Controlled Switching
}

\author{
Shengxiang Jiang and João P. Hespanha \\ Electrical and Computer Engineering \\ University of California at Santa Barbara
}

\begin{abstract}
In this paper we consider the problem of designing a multi-controller $K$ that consists of a family of stabilizing controllers $\left\{K_{m}\right\}_{m=1}^{n}$, where each controller $K_{m}$ belongs to a constrained set $\mathscr{K}_{m}$. Our goal is to determine the controllers $\left\{K_{m}\right\}_{m=1}^{n}$ (and an associated switching trajectory if switching is controllable) to optimize the closed-loop system performance. Based on a special implementation of the multicontroller, the design problem under uncontrolled switching can be transformed into a sequence of convex programs, the solutions to which provide an approximate solution to the multi-controller design problem with an arbitrary given precision; while in the case of controlled switching, a heuristic method is proposed to find a suboptimal switching signal and the corresponding multi-controller. A numerical example is presented to illustrate the efficiency of the proposed algorithms.
\end{abstract}

Keywords: multi-controller, switching, convex, optimization

\section{INTRODUCTION}

Multi-controller design has been studied for many years, and is receiving increasing attention, due to their tolerance to system failure [1], [2], flexibility in linear parametervarying applications [3], [4], and success in modeling of logic-based systems [5], [6]. Research along this line is also motivated by multi-objective design with conflicting requirements [7], [8] and control over networks [9], [10]. The main idea behind these results is as follows: in the control of complex systems, a single linear time-invariant (LTI) controller is not suitable due to the parameter-varying dynamics of the system, time-varying available information about the system, and fluctuating communication resources, hence multiple controllers must be designed, each satisfies certain specifications, and switching among them is used to achieve the overall system performance.

In this paper we consider the problem of designing a multicontroller $K$ to stabilize an LTI plant $G$. The multi-controller $K$ consists of a family of stabilizing controllers $\left\{K_{m}\right\}_{m=1}^{n}$ and an associated switching signal, where each controller $K_{m}$ belongs to the constrained set $\mathscr{K}_{m}$ and the switching signal indicates which is the operational controller within the family $\left\{K_{m}\right\}_{m=1}^{n}$. When the multi-controller switches within the family of stabilizing controllers, the resulting closed-loop system is a switched system and stability is not guaranteed, even when each controller can stabilize the plant on its own [6], [11], [12]. This framework was first studied and analyzed in [12], where system stability under arbitrary switching is guaranteed by either state reset or a special implementation of the family of controllers in state space. Alternatively, an output-switching implementation of the multi-controller in discrete time was proposed to address the effect of the steady-state performance and the transient signals due to controller switching [13], and to deal with structural constraints on the family of controllers [14].

Our goal is to design $\left\{K_{m}\right\}_{m=1}^{n}$ (and the associated switching signal if switching is controllable) to optimize the closed-loop system performance under switching. Specifications of $\mathscr{K}_{m}$ can include some performance requirement (i.e., each $K_{m}$ should achieve some performance level $\gamma_{m}$ ), a desired set of operational sensors and actuators (and hence a structural constraint on $K_{m}$ ), quality of communication service (including SNR, packet drops, and others), and so on. For different properties of the switching signal, the set of admissible switching trajectories is different, and hence the optimal achievable performance is different.

We shall consider two classes of switching options: controlled switching and uncontrolled switching. In the first class the interest is placed on synthesizing a switching signal or/and a corresponding multi-controller to stabilize the switched systems. The other is on the analysis of system stability and the synthesis of a multi-controller for a given class of switching signals, including arbitrary switching, slow switching, and stochastic switching. Uncontrolled switching might occur due to unpredictable environmental changes, information transmission loss, or component failure. Taking switching into consideration during the design stage helps preventing unacceptable system performance degradation or even loss of system stability. Based on a special implementation of the multi-controller mimicking the Youla-Kucera parametrization, the design problem under uncontrolled switching can be transformed into a sequence of convex programs, the solutions to which provide an approximate solution to the multi-controller design problem with an arbitrary given precision; while in the case of controlled switching, a heuristic method is proposed to find a suboptimal switching signal and the corresponding multicontroller.

The paper is organized as follows. In Section II we presents the basic setup and the problem formulation; we deal with the multi-controller design problem under uncontrolled switching, including arbitrary switching, slow switching, and stochastic switching in Section III and the case of controlled switching in Section IV; in Section V we present a numerical example to illustrate the efficiency of the proposed architecture and to compare the achievable performance in difference cases; we conclude in Section VI.

Notation: The Frobenius norm of a matrix $X$ with real entries, denoted by $\|X\|_{F}$ is $\sqrt{\operatorname{tr}\left(X X^{\prime}\right)} \cdot \mathcal{E}(x)$ denotes the 
expectation of a random variable (or vector) $x$. If $A$ is a matrix depending on a random vector (or process) $\xi,\|A\|_{F S}$ denotes root mean square (RMS) of its Frobenius norm, i.e., $\|A\|_{F S}=\sqrt{\mathcal{E}_{\xi}\|A\|_{F}^{2}} \cdot \mathbb{Z}^{+}$denotes the set of nonnegative integers $\{0,1,2, \ldots\}$, and $\mathbb{N}$ the set of positive integers $\{1,2, \ldots\}$. For two real numbers $\alpha$ and $\beta, \alpha \vee \beta$ denote the maximum of $\{\alpha, \beta\}$ and $\alpha \wedge \beta$ denotes the minimum of $\{\alpha, \beta\}$. For two sets $S_{1}$ and $S_{2}, S_{1} \subseteq S_{2}$ means that $S_{1}$ is a subset of $S_{2}$. For a causal linear time-varying (LTV) system $T$ with a lower (block) triangular representation

$$
T=\left[\begin{array}{cccc}
T(0,0) & & & \\
T(1,0) & T(1,1) & & \\
T(2,0) & T(2,1) & T(2,2) & \\
\vdots & \ddots & \ddots & \ddots
\end{array}\right]
$$

let $[\mathcal{R}]_{t}$ denote $[T(t, 0) \ldots T(t, t)]$, and $\left[\mathcal{M}_{T}\right]_{t}$ denote the truncated lower (block) triangular matrix

$$
\left[\begin{array}{ccc}
T(0,0) & & \\
\vdots & \ddots & \\
T(t, 0) & \cdots & T(t, t)
\end{array}\right] .
$$

The $\mathcal{H}_{2}$ norm of system $T$ with a lower (block) triangular representation (1) is defined as

$$
\|T\|_{2}=\sqrt{\sup _{t} \operatorname{tr}\left\{\left[\mathcal{R}_{T}\right]_{t}\left(\left[\mathcal{R}_{T}\right]_{t}\right)^{\prime}\right\}}=\sup _{t}\left\|\left[\mathcal{R}_{T}\right]_{t}\right\|_{F} .
$$

$\mathcal{L}_{T I}$ denotes the set of all stable LTI systems.

\section{Problem Setting}

Consider an LTI discrete-time system $G$ described as

$$
\begin{aligned}
x^{+} & =A x+B_{w} w+B u \\
z & =C_{z} x+D_{z w} w+D_{z u} u \\
y & =C x+D_{y w} w,
\end{aligned}
$$

where $x$ represents the system state, $w$ the exogenous disturbance, $u$ the control input, $z$ the regulated output, and $y$ the measurement output. We assume that $(A, B)$ is stabilizable and $(A, C)$ is detectable.

We want to design a multi-controller $u=K y$ such that the closed loop mapping of interest from $w \rightarrow z$, denoted by $^{1} \Phi(G, K)$ satisfies certain design objectives. The multicontroller $K$ consists of a set of LTI controllers $\left\{K_{m}\right\}_{m=1}^{n}$ with the property that each controller $K_{m}$ stabilizes the plant in (2) on its own and belongs to a constrained set $\mathscr{K}_{m}$. To proceed, we first introduce the switching signal $\sigma: \mathbb{Z}^{+} \rightarrow\{1,2, \ldots, n\}$, which indicates the status of the multi-controller and assumes a value $m \in\{1,2, \ldots, n\}$ at time $t$, i.e., $\sigma(t)=m$ if the multi-controller $K$ switches to $K_{m}$ at that time. Let $\Xi$ denote the set of admissible switching trajectories of $\sigma$ under consideration, which will be specified explicitly in Sections III and IV. For a specific switching trajectory $\{\sigma(t)\}_{t=0}^{\infty}$ of $\sigma \in \Xi$, let $K(\sigma)$ denote the resulting

\footnotetext{
${ }^{1} \Phi(G, K)$ denotes the closed-loop mapping emphasizing its dependence on the parameters in the parentheses, i.e., the plant $G$ and the controller $K$. Similar notation holds for $\Phi\left(G, K_{m}\right)$ and $\Phi(G, K(\sigma))$ in this paper.
}

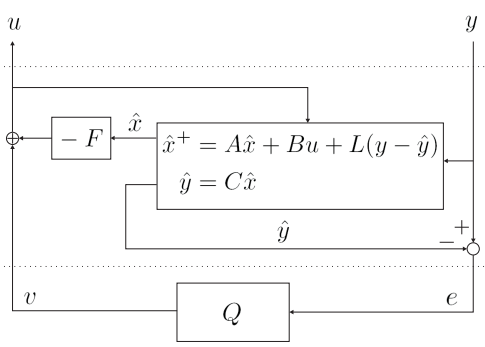

Fig. 1. Structure of all stabilizing controllers

LTV controller, which depends causally on $\sigma$. In this case, the closed-loop mapping $\Phi(G, K(\sigma))$ is a switched system.

As noted, e.g., in [6], [12], even if $\Phi\left(G, K_{m}\right)$ is stable for all $m \in\{1, \ldots, n\}$, the stability of the switched system $\Phi(G, K(\sigma))$ is not guaranteed. To overcome this challenge, we adopt a special implementation for the multicontroller based on the Youla-Kucera parametrization. Given that $(A, B)$ is stabilizable and $(A, C)$ is detectable, there exist matrices $F$ and $L$ such that $A-B F$ and $A-L C$ are Hurwitz. The set of all stabilizing controllers can be formulated via the Youla-Kucera parametrization, as stated in the following result.

Fact II.1: [15] All controllers that internally stabilize $G$ in (2) can be parameterized as

$$
\begin{aligned}
K & =(\tilde{X}-Q \tilde{N})^{-1}(\tilde{Y}-Q \tilde{M}) \\
& =(Y-M Q)(X-N Q)^{-1},
\end{aligned}
$$

where $Q$ is the (LTI) Youla parameter and stable; and all such controllers can be implemented using the structure in Figure 1. Moreover, the closed-loop mapping of interest $\Phi(G, K)=$ $H-U Q V$ is affine in $Q$.

For details about the dynamics of $X, \tilde{X}, Y, \tilde{M}, \tilde{M}$, $N, \tilde{N}, H, U$ and $V$, see [15]. Based on Fact II.1, there exists a corresponding Youla parameter $Q_{m}$ such that

$$
\begin{aligned}
K_{m} & =\left(\tilde{X}-Q_{m} \tilde{N}\right)^{-1}\left(\tilde{Y}-Q_{m} \tilde{M}\right) \\
& =\left(Y-M Q_{m}\right)\left(X-N Q_{m}\right)^{-1} .
\end{aligned}
$$

It is easy to show that

$$
\begin{aligned}
Q_{m} & =\left(\tilde{Y}-\tilde{X} K_{m}\right)\left(\tilde{M}-\tilde{N} K_{m}\right)^{-1} \\
& =\left(M-K_{m} N\right)^{-1}\left(Y-K_{m} X\right) .
\end{aligned}
$$

Therefore, constraints on the controller $K_{m}$ can be transformed equivalently into the constraints on the Youla parameter $Q_{m}$. Let $\mathscr{Q}_{m}$ denote the possible constraints on $Q_{m}$ such that $K_{m} \in \mathscr{K}_{m}$ if and only if $Q_{m} \in \mathscr{Q}_{m}$. For each stabilizing controller $K_{m}$, if we keep the state feedback gain $F$ and the observer gain $L$ in Figure 1 the same for every controller $K_{m}$, and switch only the Youla parameter $Q$ to $Q_{m}$, the linear mapping from $\left[\begin{array}{ll}w^{\prime} & v^{\prime}\end{array}\right]^{\prime} \rightarrow\left[\begin{array}{ll}z^{\prime} & e^{\prime}\end{array}\right]^{\prime}$ as shown in Figure 2, can be kept constant and equal to

$$
\left[\begin{array}{cc}
H & -U \\
V & 0
\end{array}\right]
$$

where $v$ is part of the control signal, $\hat{x}$ denotes the state estimation, $e$ is the estimation residual $y-C \hat{x}$, and $H, U, V$ are provided in Fact II.1. 


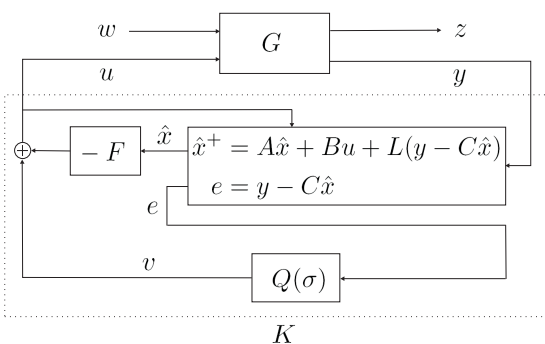

Fig. 2. Structure of the multi-controller $K$

We thus adopt the multi-controller structure in Figure 2, where $Q(\sigma)$ is the switched system depending causally on the switching signal $\sigma$, which switches among the family $\left\{Q_{m}\right\}_{m=1}^{n}$. The closed-loop mapping from $w \rightarrow z$, denoted by $\Phi(G, K(\sigma))$ equals $H-U Q(\sigma) V$. Since the systems $H, U$, and $V$ remain constant and stable, $\Phi(G, K(\sigma))$ is bounded-input bounded-output (BIBO) stable under switching if and only if $Q(\sigma)$ is BIBO stable under switching. To guarantee the system stability under switching, we adopt the implementation of $Q(\sigma)$ with an input-output description as

$$
\left[\begin{array}{cccc}
Q_{\sigma(0)}(0) & & & \\
Q_{\sigma(1)}(1) & Q_{\sigma(1)}(0) & & \\
Q_{\sigma(2)}(2) & Q_{\sigma(2)}(1) & Q_{\sigma(2)}(0) & \\
\vdots & \ddots & \ddots & \ddots
\end{array}\right]
$$

where $Q_{\sigma(t)}(k)=Q_{m}(k)$ if $\sigma(t)=m, \forall t \geq 0 \forall k \geq 0$, and $\left\{Q_{m}(k)\right\}_{k=0}^{\infty}$ denote the unit impulse response of the LTI system $Q_{m}$. Let $Q_{\sigma}$ denote the switched system with an input-output description in (4). The novel properties of the above implementation are that (i) the output $v(t)(t \geq 0)$ depends only on the switching signal at time $t$, i.e. $\sigma(t)$, and (ii) BIBO stability under switching is guaranteed since

$$
\left\|Q_{\sigma}\right\|_{2}=\sup _{t}\left\|\left[Q_{\sigma(t)}(t) \ldots Q_{\sigma(t)}(0)\right]\right\|_{F} \leq \max _{1 \leq m \leq n}\left\|Q_{m}\right\|_{2}
$$

is independent of the switching signal. This implementation was also considered in [13], [14] in different setups and different performance criteria.

Since $e=V w$ and $v=Q_{\sigma} e$, it follows that ${ }^{2}$

$$
\|w \rightarrow v\|_{2}=\left\|Q_{\sigma} V\right\|_{2} \leq \max _{1 \leq m \leq n}\left\|Q_{m} V\right\|_{2} .
$$

Using the fact that $z=H w-U v$, we can infer that

$$
\begin{aligned}
\|w \rightarrow z\|_{2} & \stackrel{(\mathrm{a})}{\leq}\|H\|_{2}+\|w \rightarrow(U v)\|_{2} \\
& \stackrel{(\mathrm{b})}{\leq}\|H\|_{2}+\|U\|_{\infty}\|w \rightarrow v\|_{2} \\
& \stackrel{(\mathrm{c})}{\leq}\|H\|_{2}+\|U\|_{\infty} \max _{1 \leq m \leq n}\left\|Q_{m} V\right\|_{2},
\end{aligned}
$$

which means that the BIBO stability of the closed-loop system is guaranteed under switching. Here inequality (a) follows from the triangle inequality of the norm, (b) is based on the facts that the norm of a composition operator is

\footnotetext{
${ }^{2}$ For simplicity of notation, $w \rightarrow v$ denotes the linear mapping from $w$ to $v$. Similar notation holds for $w \rightarrow z, w \rightarrow(U v)$, etc.
}

upper bounded by the multiplicative product of individual operator's norms and that the $\mathcal{H}_{\infty}$ norm of a linear operator equals the induced norm of input-output power (RMS value) [15], and (c) is shown in (5).

In the sequel, we consider the performance optimization of the closed-loop system $H-U Q_{\sigma} V$ under switching. The precise design problem is stated as follows.

Find the conditions for the existence of a multi-controller $K$ with $n$ controllers $\left\{K_{m}\right\}_{m=1}^{n}$ (and an associated switching trajectory if switching is controllable) such that $K_{m} \in$ $\mathscr{K}_{m}, \forall m \in\{1,2, \ldots, n\}$ and the closed-loop system performance $\|\Phi(G, K)\|=\left\|H-U Q_{\sigma} V\right\|$ is optimized.

The interpretation of $\left\|H-U Q_{\sigma} V\right\|$ depends on the property of $\sigma$, as explored in Section III and IV. We make the following assumption to make the final formulations convex.

Assumption II.1: For any $m \in\{1, \ldots, n\}$, the design problem $\inf _{K_{m} \in \mathscr{K}_{m}}\left\|\Phi\left(G, K_{m}\right)\right\|$ can be formulated equivalently via a convex program in $Q_{m}$.

The meaning of the assumption is that without switching, the design problem to obtain an optimal controller in $\mathscr{K}_{m}$ can be transformed into a convex program and solved completely. Most centralized design problems and some design problems in decentralized control fit here; for example, see [16], [17]

\section{Control Under UnCONTROLlEd SWitching}

In this section, we consider the multi-controller design problem under uncontrolled switching. The design problem is to find LTI systems $\left\{Q_{m}\right\}_{m=1}^{n}$ solving

$$
\nu(\Xi):=\inf _{\left\{Q_{m} \in \mathscr{Q}_{m}\right\}_{m=1}^{n}}\left\|H-U Q_{\sigma} V\right\| .
$$

\section{A. Control under arbitrary switching}

First, we consider the case in which the switching signal $\sigma$ can assume an arbitrary value in $\{1, \ldots, n\}$ at any time, i.e., the set of admissible switching trajectories is $\Xi_{1}=\{\sigma \mid \forall t \geq$ $0, \sigma(t) \in\{1, \ldots, n\}\}$. The worst-case $\mathcal{H}_{2}$ norm criterion is adopted, i.e., $\left\|H-U Q_{\sigma} V\right\|=\sup _{\sigma \in \Xi_{1}}\left\|H-U Q_{\sigma} V\right\|_{2}$. In general, it is difficult or even impossible to determine $\nu\left(\Xi_{1}\right)$ exactly. To overcome this difficulty, we formulate two sequences of quadratic programs with increasing complexity, the solutions to which provide two sequences converging to the optimal performance $\nu\left(\Xi_{1}\right)$ from below and from above respectively, as stated in Theorem 3.1.

Towards this goal, define for each $i \in \mathbb{Z}^{+}$

$$
\begin{aligned}
& \underline{\nu}^{i}=\inf _{\left\{Q_{m} \in \mathscr{Q}_{m}\right\}_{m=1}^{n}} \sup _{1 \leq t \leq i} \sup _{\sigma \in \Xi_{1}}\left\|\left[\mathcal{R}_{H-U Q_{\sigma} V}\right]_{t}\right\|_{F}, \\
& \bar{\nu}^{i}=\inf _{\left\{\begin{array}{c}
Q_{m} \in \mathscr{Q}_{m} \\
Q_{m}(l)=0 \forall l>i
\end{array}\right\}_{m=1}^{n} \sup _{m} \sup _{\sigma \in \Xi_{1}}\left\|\left[\mathcal{R}_{H-U Q_{\sigma} V}\right]_{t}\right\|_{F}} .
\end{aligned}
$$

In the definition of $\underline{\nu}^{i}$ the optimizing objective is the truncated input-output mapping of $H-U Q_{\sigma} V$ over time $[0, i]$, and the minimizing parameters are constrained to FIR filters of order $i$ in the definition of $\bar{\nu}^{i}$. It is easy to show that $\underline{\nu}^{i} \leq \underline{\nu}^{i+1} \leq \nu^{o}\left(\Xi_{1}\right)$, and $\bar{\nu}^{i} \geq \bar{\nu}^{i+1} \geq \nu^{o}\left(\Xi_{1}\right), \forall i$, where

$$
\nu^{o}\left(\Xi_{1}\right)=\inf _{\left\{Q_{m} \in \mathscr{Q}_{m}\right\}_{m=1}^{n}} \sup _{t} \sup _{\sigma \in \Xi_{1}}\left\|\left[\mathcal{R}_{H-U Q_{\sigma} V}\right]_{t}\right\|_{F} .
$$

First, we introduce the following results. 
Lemma III-A.I: $\nu\left(\Xi_{1}\right)=\nu^{o}\left(\Xi_{1}\right)$.

Lemma III-A.2: $\left\{\sup _{\sigma \in \Xi_{1}}\left\|\left[\mathcal{R}_{H-U Q_{\sigma} V}\right]_{t}\right\|_{F}\right\}_{t=0}^{\infty}$ is a monotonically increasing sequence in $t$, for any given systems $\left\{Q_{m}\right\}_{m=1}^{n}$.

Similar results to Lemma III-A.1 and III-A.2 appear in [14], and hence the proof details are omitted here. Given the fact in Lemma III-A.2, the lower and upper bound sequences can be simplified to

$$
\begin{aligned}
& \underline{\nu}^{i}=\inf _{\left\{Q_{m} \in \mathscr{Q}_{m}\right\}_{m=1}^{n}} \sup _{\sigma \in \Xi_{1}}\left\|\left[\mathcal{R}_{H-U Q_{\sigma} V}\right]_{i}\right\|_{F}, \\
& \left.\bar{\nu}^{i}=\inf _{\substack{Q_{m} \in \mathcal{Q}_{m} \\
Q_{m}(l)=0, \forall l>i}}\right\}_{m=1}^{n} \lim _{t \rightarrow \infty} \sup _{\sigma \in \Xi_{1}}\left\|\left[\mathcal{R}_{H-U Q_{\sigma} V}\right]_{t}\right\|_{F} .
\end{aligned}
$$

Let $\Phi^{i}=\left[\Phi_{i}^{i} \Phi_{i-1}^{i} \ldots \Phi_{0}^{i}\right]$ denote $\left[\mathcal{R}_{H-U Q_{\sigma} V}\right]_{i}$, then

$$
\Phi_{k}^{i}=H(k)-\sum_{m=0}^{k} U(m) \sum_{l=0}^{k-m} Q_{\sigma(i-m)}(l) V(k-m-l),
$$

$\forall k \in\{0,1, \ldots, i\}$. In general, $\sup _{\sigma \in \Xi_{1}}\left\|\Phi^{i}\right\|$ is a convex program depending on all the $n^{i+1}$ switching trajectories of $\{\sigma(t)\}_{t=0}^{i}$, which could be computationally intractable since $n^{i+1}$ tends exponentially to $\infty$ as $i \rightarrow \infty$. In several control applications, $U$ can be chosen to be a FIR filter of order $r$ at most equal to the dimension of the system to be controlled. More generally, since $U$ is stable and in $\mathcal{L}_{T I}$, it can always be approximated by a FIR filter. In this case the complexity is manageable, as we indicate in what follows.

If there exists a finite $r$ such that $U(k)=0$ for all $k>r$, then $\forall k \in\{0,1, \ldots, i\}$

$$
\Phi_{k}^{i}=H(k)-\sum_{m=0}^{k \wedge r} U(m) \sum_{l=0}^{k-m} Q_{\sigma(i-m)}(l) V(k-m-l)
$$

and $\Phi^{i}$ will depend on the switching signal at (at most) $r+1$ switching instants: $\{\sigma(t)\}_{t=0 \vee(i-r)}^{i}$, which means that we only need to concentrate on the switching signal over $[0 \vee(i-r), i]$, i.e., we need to consider at most $n^{r+1}$ switching sequences. Then the problem of determining $\underline{\nu}^{i}$ and $\bar{\nu}^{i}$ can be determined in polynomial time since there are at most $n^{r+1}$ norm constraints, which is independent of $i$. More explicitly, we can formulate two sequences of convex programs determining $\left\{\underline{\nu}^{i}\right\}_{i=0}^{\infty}$ and $\left\{\bar{\nu}^{i}\right\}_{i=0}^{\infty}$ as follows.

For given $i, \Phi^{i}$, and hence $\underline{\nu}^{i}$ depend only on $\left\{Q_{m}(l)\right\}_{m=1}^{n}, l=0, \ldots, i$. The optimization problem is:

$$
\begin{aligned}
& \underline{\nu}^{i}=\inf _{\left\{Q_{m}(l)\right\}_{m=1}^{n}, l=0, \ldots i,} \gamma \\
& Q_{m} \in \mathscr{Q}_{m}, \forall m \in\{1, \ldots, n\}, \\
& \gamma \geq\left\|\left[\Phi_{i}^{i}, \Phi_{i-1}^{i}, \ldots, \Phi_{0}^{i}\right]\right\|, \forall \sigma(t) \in\{1, \ldots, n\} \\
& \quad \text { for } t=0 \vee(i-r), \ldots, i,
\end{aligned}
$$

where $\left\{\Phi_{k}^{i}\right\}_{k=0}^{i}$ are provided in (7).

If $\left\{Q^{m}(l)\right\}_{m=1}^{n}=0, \forall l>i$ and there exists a finite $\tau$ such that $V(k)=0$ for all $k>\tau$, then there are $r+i+\tau+1$ (block) entries of $\Phi^{t}$ depending on the nonzero parameters $\left\{Q_{m}(l)\right\}_{m=1}^{n}, l \leq i$, i.e., $\Phi_{0}^{t}, \ldots, \Phi_{r+i+\tau}^{t}$ when $t \geq r+i+\tau$.
Then for a given $t \geq r+i+\tau$, the upper bound $\bar{\nu}^{i}$ is determined as:

$$
\begin{aligned}
& \bar{\nu}^{i}=\inf _{\left\{Q_{m}(l)\right\}_{m=1}^{n}, l=0, \ldots, i} \gamma \\
& Q_{m} \in \mathscr{Q}_{m}, \forall m \in\{1, \ldots, n\} \\
& \gamma \geq\left\|\left[b, \Phi_{r+i+\tau}^{t}, \ldots, \Phi_{0}^{t}\right]\right\|, \forall \sigma(k) \in\{1, \ldots, n\} \\
& \quad \text { for } k=(t-r), \ldots, t .
\end{aligned}
$$

where $\Phi_{k}^{t}=H(k)-\sum_{m=0 \vee(k-i-\tau)}^{k \wedge r} U(m)$

$$
\begin{aligned}
& \times \sum_{t=0 \vee(k-m-\tau)}^{(k-m) \wedge i} Q_{\sigma(t-m)}(t) V(k-m-t), \forall k \leq r+i+\tau \\
& b\left(m_{1}\right)=\sqrt{\sum_{k=r+i+\tau+1}^{\infty} \sum_{m_{2}=1}^{n_{c}}\left|(H(k))_{m_{1} m_{2}}\right|^{2}} \\
& b=\left[b(1) \ldots b\left(n_{r}\right)\right]^{\prime} .
\end{aligned}
$$

Here $n_{r}, n_{c}$ are the dimensions of the output and input of the LTI system $H$ respectively. The solution to problem (9) is independent of $t$ as long as $t>r+i+\tau$.

Following the proofs of Theorem 6.1 and 6.2 in [16], we can show that $\bar{\nu}^{i} \searrow \nu\left(\Xi_{1}\right), \underline{\nu}^{i} \nearrow \nu\left(\Xi_{1}\right)$, as $i \rightarrow \infty$. Summarizing we have

Theorem 3.1: The worst-case $\mathcal{H}_{2}$ optimization problem (6) under arbitrary switching is solvable when $U$ and $V$ are FIR filters, and an approximate solution with any given precision can be determined by solving two sequences of convex programs in (8) and (9).

\section{B. Control under slow switching}

In this subsection, we consider the case that the operational duration of each controller is lower bounded. For any $t \geq 0$, let $\ell(\sigma(t))$ denote the length of the shortest consecutive switching sequence $\left\{\sigma\left(t_{1}\right), \sigma\left(t_{1}+1\right), \ldots \sigma\left(t_{2}\right)\right\}$ with all values equal to $\sigma(t)$ and $t_{1} \leq t \leq t_{2}, \Xi_{2}^{s}$ the set of admissible switching trajectories $\{\sigma \mid \forall t \geq 0, \ell(\sigma(t)) \geq s$.$\} , and \Xi_{2}^{s, t}$ the maximum set of unique switching sequences over a time period of $t$ drawn from $\Xi_{2}^{s}, \forall s \in \mathbb{N}$.

The performance criterion adopted is that given any $s \in$ $\mathbb{N},\left\|H-U Q_{\sigma} V\right\|=\sup _{\sigma \in \Xi_{2}^{s}}\left\|H-U Q_{\sigma} V\right\|_{2}$. Note that $\Xi_{2}^{s+1} \subseteq \Xi_{2}^{s}$ and $\Xi_{1}=\Xi_{2}^{1}$, it follows that $\nu\left(\Xi_{1}\right) \geq \nu\left(\Xi_{2}^{s}\right)=$ $\nu\left(\Xi_{2}^{s+1}\right)$ due to the worst-case performance criterion. A helpful inequality is that $\nu\left(\Xi_{1}\right) \geq \nu\left(\Xi_{2}^{s}\right) \geq \nu\left(\Xi_{2}^{\infty}\right) \geq$ $\max _{m} \inf _{Q_{m} \in \mathscr{Q}_{m}}\left\|H-U Q_{m} V\right\|_{2}$, i.e., the worst-case performance under arbitrary or slow switching can not be better than the worst-case (steady) performance without switching.

Similar to the case of control under arbitrary switching, we can formulate two sequences of convex programs converging to the optimal performance from below and above respectively. In the lower bound sequence we need to replace $\forall \sigma(t) \in\{1, \ldots, n\}$ for $t=0 \vee(i-r), \ldots, i$ in the design problem (8) with $\forall\{\sigma(0 \vee(i-r)), \ldots, \sigma(i)\} \in$ $\Xi_{2}^{s,(i+1) \wedge(r+1)}$; while in the upper bound sequence $\forall \sigma(k) \in$ $\{1, \ldots, n\}$ for $k=(t-r), \ldots, t$ in (9) should be replaced by 
$\forall\{\sigma(t-r), \ldots, \sigma(t)\} \in \Xi_{2}^{s, r+1}$. In any case, the maximum number of norm constraints will decrease from $n^{r+1}$ to the cardinality of the set $\left|\Xi_{2}^{s, r+1}\right|$, which equals $n+r n(n-1)$ if $s \geq r+1$. If $s<r+1$, determining $\left|\Xi_{2}^{s, r+1}\right|$ is more involved, but its value is upper bounded by $n^{r+1}$.

\section{Control under stochastic switching}

Last, we consider the case that the transition of the switching signal $\sigma$ occurs according to some Markov chains, and adopt the stochastic average $\mathcal{H}_{2}$ norm as the performance criterion, i.e., $\left\|H-U Q_{\sigma} V\right\|=\sup _{t} \sqrt{\mathcal{E}_{\sigma}\left\|\left[\mathcal{R}_{H-U Q_{\sigma} V}\right]_{t}\right\|_{F}^{2}}$.

We assume that the switching signal $\sigma$ is driven by a Markov chain with a probability transition matrix $P$. Let $\pi=\left[\pi_{1}, \ldots, \pi_{n}\right]$ denote the stationary probability distribution such that $\pi=\pi P$, and $\Xi_{3}$ the corresponding set of admissible switching trajectories. For an arbitrary switching sequence $\left.\left\{\sigma\left(t_{0}\right)\right), \ldots, \sigma\left(t_{i}\right)\right\}$ of length $i+1$, its occurrence probability, denoted by $P\left(\left\{j_{0}, \ldots, j_{i}\right\}\right)$ equals $\pi_{j_{0}} P_{j_{0} j_{1}} \ldots P_{j_{i-1} j_{i}}$, where $j_{k}=\sigma\left(t_{k}\right) \forall k \in\{0, \ldots, i\}$.

Similar to the case of arbitrary switching in Section III-A, define for each $i \in \mathbb{Z}^{+}$

$$
\begin{aligned}
\underline{\nu}_{s}^{i} & =\inf _{\left\{Q_{m} \in \mathscr{Q}_{m}\right\}_{m=1}^{n}} \sup _{i}\left\|\left[\mathcal{R}_{H-U Q_{\sigma} V}\right]_{i}\right\|_{F S}, \\
\bar{\nu}_{s}^{i} & \left.=\inf _{\substack{Q m \in \mathcal{Q} m \\
Q_{m}(l)=0 \\
\forall l>i}}\right\}_{m=1}^{n}=0 \sup \left\|\left[\mathcal{R}_{H-U Q_{\sigma} V}\right]_{t}\right\|_{F S},
\end{aligned}
$$

which converge to the optimal performance $\nu\left(\Xi_{3}\right)$ from below and above respectively. More specifically, the lower bound $\underline{\nu}_{s}^{i}$ is defined as

$$
\begin{aligned}
\underline{\nu}_{s}^{i}= & \inf _{\left\{Q_{m}\right\}_{m=1}^{n}} \sqrt{\sum_{j_{\mathrm{O} \vee(i-r)}, \ldots, j_{i}} P\left(\left\{j_{0 \vee(i-r)}, \ldots, j_{i}\right\}\right) \gamma_{j_{\mathrm{O} \vee(i-r)} \ldots j_{i}}^{2}} \\
& Q_{m} \in \mathscr{Q}_{m}, \forall m \in\{1, \ldots, n\} \\
& \gamma_{j_{0 \vee(i-r)} \ldots j_{i}} \geq\left\|\Phi^{i}\right\|_{F}, \sigma(k)=j_{k} \\
& \quad \text { for } k=0 \vee(i-r), \ldots, i
\end{aligned}
$$

where $j_{0 \vee(i-r)}, \ldots, j_{i} \in\{1, \ldots, n\}$, and $\Phi^{i}$ is defined in Section III-A. For a given $t \geq r+i+\tau$, the upper bound $\bar{\nu}_{s}^{i}$ is determined as

$$
\begin{gathered}
\bar{\nu}_{s}^{i}=\inf _{\substack{\left\{Q_{m}(l)\right\}_{m}^{n}=1 \\
l=0, \ldots, i}} \sqrt{\sum_{j_{t-r}, \ldots, j_{t}} P\left(\left\{j_{t-r}, \ldots, j_{t}\right\}\right) \bar{\gamma}_{j_{t-r} \ldots j_{t}}^{2}} \\
Q_{m} \in \mathscr{Q}_{m}, \forall m \in\{1, \ldots, n\} \\
\bar{\gamma}_{j_{t-r} \ldots j_{t}} \geq\left\|\left[b, \Phi_{r+i+\tau}^{t}, \ldots, \Phi_{0}^{t}\right]\right\|_{F}, \forall \sigma(k) \in\{1, \ldots, n\} \\
\text { for } k=t-r, \ldots, t,
\end{gathered}
$$

where $\Phi_{k}^{t}$ and $b$ are defined in (7) and (9) respectively.

\section{Remark}

In the first two cases we adopt the worst-case (deterministic) performance; while in the third we consider optimizing the stochastic average performance. Since all the problem formulations are convex in the design parameters, it is feasible to combine the three cases into a unified design framework, and to analyze the interplay among the local steady performance without switching, the worst-case performance, and the stochastic performance.

\section{Control Under Controlled Switching}

In this section we consider the multi-controller design problem under controlled switching, i.e., now the design problem is to find LTI systems $\left\{Q_{m}\right\}_{m=1}^{n}$ and a switching trajectory of $\sigma$ solving the following optimization problem

$$
\nu\left(\Xi_{4}\right):=\inf _{\sigma \in \Xi_{4},\left\{Q_{m} \in \mathscr{Q}_{m}\right\}_{m=1}^{n}}\left\|H-U Q_{\sigma} V\right\|,
$$

where $\left\|H-U Q_{\sigma} V\right\|=\left\|H-U Q_{\sigma} V\right\|_{2}$.

If we allow arbitrary switching, e.g., $\Xi_{4}=\Xi_{1}$, and there is an integer $m \in\{1,2, \ldots, n\}$ such that

$$
\inf _{Q_{m} \in \mathscr{Q}_{m}}\left\|H-U Q_{m} V\right\|=\inf _{Q^{o} \in \mathcal{L}_{T I}}\left\|H-U Q^{o} V\right\|,
$$

the trivial solution is to set $\sigma(t)=m, \forall t \geq 0$ and $Q_{m}=$ $Q^{o}$. So, proper specifications on the admissible switching sequences in $\Xi_{4}$ is necessary to balance the performance requirement and resource utilization. In general, the design problem in (12) is not convex due to the requirement that $\sigma$ must belong to the set $\Xi_{4}$. To reduce the computational complexity, we restrict out attention to periodic switching trajectories in $\Xi_{4}$. Let $\Xi_{4}^{N}$ denote the following subset of admissible switching trajectories $\left\{\sigma \mid \sigma \in \Xi_{4}, \sigma(t)=\sigma(t+\right.$ N) $\forall t \geq 0\}$, where $N \in \mathbb{N}$.

Given a switching trajectory $\sigma \in \Xi_{4}^{N}$ and Youla parameters $\left\{Q_{m}\right\}_{m=1}^{n}$, the sequence $\left\{\left\|\left[\mathcal{R}_{H-U Q_{\sigma} V}\right]_{t}\right\|_{F}\right\}_{t=0}^{\infty}$ is not necessarily monotonically increasing or periodic, but it holds that $\left\|\left[\mathcal{R}_{H-U Q_{\sigma} V}\right]_{t}\right\|_{F} \leq\left\|\left[\mathcal{R}_{H-U Q_{\sigma} V}\right]_{N+t}\right\|_{F}, \forall t \geq 0$ since $\left[\Phi_{t}^{N+t}, \ldots, \Phi_{0}^{N+t}\right]=\left[\mathcal{R}_{H-U Q_{\sigma} V}\right]_{t}$. This means that

$$
\max _{0 \leq t \leq i}\left\|\left[\mathcal{R}_{H-U Q_{\sigma} V}\right]_{t}\right\|_{F} \leq \max _{O \vee(i-N+1) \leq t \leq i}\left\|\left[\mathcal{R}_{H-U Q_{\sigma} V}\right]_{t}\right\|_{F}
$$

and hence the design problem can be transformed to

$$
\nu\left(\Xi_{4}^{N}\right)=\inf _{\substack{\sigma \in \Xi_{4}^{N} \\\left\{Q_{m} \in \mathcal{Q}_{m}\right\}_{m=1}^{n}}} \lim _{i \rightarrow \infty} \max _{\operatorname{ov}(i-N+1) \leq t \leq i}\left\|\left[\mathcal{R}_{H-U Q_{\sigma} V}\right]_{t}\right\|_{F}
$$

For each given periodic switching signal $\sigma$, the above design problem can be solved efficiently via convex programs as before to find $\left\{Q_{m}\right\}_{m=1}^{n}$. However, it is still computationally expensive to find an optimal solution of a periodic switching signal and associated Youla parameters. Instead of an exhaust search, we adopt a heuristic search method from [9].

1) Set $s_{0}=k^{o}$ if $Q_{k^{o}}$ achieves the best performance in the set $\left\{\inf _{Q_{k} \in \mathscr{Q}_{k}}\left\|H-U Q_{k} V\right\|_{2}\right\}_{k=1}^{n}$.

2) Assume that the suboptimal switching sequence of length $i$ is $s_{i-1}$ obtained at stage $i-1$, the switching signal $\sigma_{i-1}$ is an infinite repetition of $s_{i-1}$, and the corresponding system performance is $\gamma_{i-1}^{o}$. Formulate a new switching sequence $s_{i}=\left\{s_{i-1}, k\right\}$ and the corresponding periodic switching signal $\sigma_{i}=\left\{s_{i}, s_{i}, \ldots\right\}$, choose the best value of $k \in\{1, \ldots, n\}$ solving

$$
\inf _{\left\{Q_{m} \in \mathscr{Q}_{m}\right\}_{m=1}^{n}}\left\|H-U Q_{\sigma_{i}} V\right\|_{2} \text { s.t. } \sigma_{i} \in \Xi_{4}^{i+1},
$$

and let $\gamma_{i}^{o}$ denote the corresponding performance.

3) If $\gamma_{i}^{o}<\gamma_{i-1}^{o}-\epsilon$ for some $\epsilon>0$, set $i=i+1$ and go back to step 2); otherwise, stop the iteration. The suboptimal switching signal is $\sigma_{i}$ if $\gamma_{i}^{o}<\gamma_{i-1}^{o}$ or $\sigma_{i-1}$ 
if $\gamma_{i}^{o} \geq \gamma_{i-1}^{o}$. The corresponding Youla parameters can be determined as the solution to (13).

The above iterative algorithm is sure to terminate because the system performance will decrease unless the iteration stops and the achievable performance is lower bounded by 0 . To obtain a suboptimal switching signal of period $N$, the proposed heuristic method needs to solve problem (13) (at most) $n N$ times.

\section{NumERICAL EXAMPLE}

In this section, we consider a simple numerical example with 1 measurement output and 2 control inputs, as follows.

$$
\begin{aligned}
x^{+}= & {\left[\begin{array}{cc}
1.2698 & 0.1352 \\
-0.8960 & -0.1390
\end{array}\right] x+\left[\begin{array}{c}
-1.1634 \\
1.1837
\end{array}\right] w } \\
& +\left[\begin{array}{cc}
-0.0154 & -0.7164 \\
0.5362 & -0.6556
\end{array}\right] u \\
z= & {\left[\begin{array}{cc}
0.3144 & 1.8482 \\
0.1068 & -0.2751
\end{array}\right] x+\left[\begin{array}{l}
2.2126 \\
1.5085
\end{array}\right] w } \\
& +\left[\begin{array}{cc}
-1.9451 & -0.5735 \\
-1.6805 & -0.1858
\end{array}\right] u \\
y= & {\left[\begin{array}{ll}
0.0089 & 0.8369
\end{array}\right] x-0.7223 w, }
\end{aligned}
$$

$F$ and $L$ can be chosen such that $r=1$ and $\tau=2$. We will consider the case of $n=2$, where specifications in $\left\{\mathscr{Q}_{m}\right\}_{m=1}^{2}$ are as follows:

$$
\begin{aligned}
& \mathscr{Q}_{1}=\left\{Q \mid Q \in \mathcal{L}_{T I},\left[\begin{array}{ll}
0 & 1
\end{array}\right] Q=0\right\}, \\
& \mathscr{Q}_{2}=\left\{Q \mid Q \in \mathcal{L}_{T I},\|H-U Q V\|_{2} \leq 6\right\} .
\end{aligned}
$$

Without switching, we can determine the optimal system performance for each family of stabilizing controllers as

$$
\begin{aligned}
& \gamma_{1}^{o}:=\inf _{Q_{1} \in \mathscr{Q}_{1}}\left\|H-U Q_{1} V\right\|_{2} \simeq 9.7573, \\
& \gamma_{2}^{o}:=\inf _{Q_{2} \in Q_{2}}\left\|H-U Q_{2} V\right\|_{2} \simeq 5.1943 .
\end{aligned}
$$

A. Arbitrary switching: The optimization problem in (6) can be solved by the algorithm proposed in Section III-A with an optimal performance $\nu\left(\Xi_{1}\right) \simeq 17.0506$, which is greater than $\gamma_{1}^{o} \vee \gamma_{2}^{o}=9.7573$ and achieved by two FIR filters $Q_{1}$ and $Q_{2}$ of order 8.

B. Slow switching: Since $r=1$ and $\Xi_{2}^{1, r+1}=\Xi_{2}^{s, r+1}$ for any finite $s \in \mathbb{N}$, there is no performance difference between arbitrary switching and slow switching except the degenerate case of no switching $\Xi_{2}^{\infty}$, i.e., $\nu\left(\Xi_{2}^{s}\right) \simeq 17.0506,1 \leq s<$ $\infty, \nu\left(\Xi_{2}^{\infty}\right)=9.7573$.

C. Stochastic switching: We assume that $P(\sigma(t)=m)=$ $0.5, \forall t \geq 0 \forall m \in\{1,2\}$. In this case the optimal performance $\nu\left(\Xi_{3}\right)$ is equal to 11.9953 approximately, achieved by two FIR filters $Q_{1}$ and $Q_{2}$ of order 8 .

D. Controlled switching: To avoid the trivial case that $K_{2}$ is operational all the time, we concentrate on the case where each controller is operational at least once for $\kappa$ consecutive time steps, i.e. the set of admissible switching trajectories is $\Xi_{4}=\left\{\sigma \mid m \in\left\{\sigma\left(t_{1}\right), \ldots, \sigma\left(t_{\kappa}\right), \forall m\{1, \ldots, n\}, \forall t_{1} \geq 0\right\}\right.$. Since $\gamma_{1}^{o}>\gamma_{2}^{o}$, in the initial step we set $s_{0}=2$. In the second step, we have to choose $s_{1}=\{2,1\}$ to avoid the case that $K_{2}$ is operational all the time; the corresponding switching signal $\sigma_{1}=\left\{s_{1}, s_{1}, \ldots,\right\}$ and the optimal system performance is 8.0172. Next, for the two switching sequences of length 3: $\left\{s_{1}, 1\right\}$ and $\left\{s_{1}, 2\right\}$, the optimal performance is 16.5816 and 12.5782 respectively, both greater than the optimal performance achieved by $\sigma_{1}$. So the proposed heuristic method stops and results in a switching signal of period 2 .

\section{CONCLUSIONS}

In this paper we considered the multi-controller design under uncontrolled and controlled switching. Through a special implementation of the multi-controller, the design problem can be translated into two sequences of convex programs converging to the optimal performance from above and below respectively in the case of uncontrolled switching. In the case of controlled switching, we relaxed the design problem to find a family of LTI parameters and an associated periodic switching sequence.

\section{REFERENCES}

[1] D. D. Siljak, "Reliable control using multiple control systems," International Journal of Control, Vol. 31, No. 2, pp. 203-329, 1980.

[2] M. Vidyasagar and N. Viswanadhan, "Reliable stabilization using a multi-controller configuration," Automatica, Vol. 21, No. 5, pp. 599602, 1985.

[3] K. J. Astrom and B. Wittenmark, Adaptive Control, 2nd Edition, Prentice Hall, December 31, 1994.

[4] A. Paul and M. G. Safonov, "Model reference adaptive control using multiple controllers and switching," Proceedings of the 42nd IEEE Conference on Decision and Control, December 2003.

[5] J.P. Hespanha and A. S. Morse, "Towards the high performance control of uncertain process via supervision," Proceedings of the 30th Annual Conference on Information Sciences and Systems, pp. 405-410, 1996

[6] D. Liberzon, Switching in Systems and Control, Birkhäuser, Boston, MA, Jun 2003.

[7] S.P. Boyd and C.H. Barrat, Linear Controller Design: Limits of Performance, New Jersey: Prentice Hall, 1991.

[8] J.C. Geromel, G. S. Deaecto, and P. Colaneri, "Multiobjective $\mathcal{H}_{2}$ control via switched linear systems," 3rd IFAC Symposium on System, Structure and Control, October 2007.

[9] L. Lu, L. Xie, and M. Fu, "Optimal control of networked systems with limited communication: a combined heuristic search and convex optimization approach,"Proceedings of the 42nd IEEE conference on Decision and Control, Vol. 2, pp. 1194-1199, December 2003.

[10] J. P. Hespanha, P. Naghshtabrizi, and Y. Xu, "A survey of recent results in networked control systems," Proceedings of IEEE Special Issue on Technology of Networked Control Systems, Vol. 95, No. 1, pp. 138 161, January 2007.

[11] R. A. DeCarlo, M. S. Branicky, S. Pettersson, and B. Lennartson, "Perspectives and results on the stability and stabilizability of hybrid systems," Proceedings of the IEEE, No. 88, No. 7, pp. 1069-1082, July 2000.

[12] J.P. Hespanha and A.S. Morse,"Switching between stabilizing controllers," Automatica, Vol. 38, No. 11, pp. 1905-1917, November 2002.

[13] G. E. Stewart and G. A. Dumont, "Finite horizon based switching between stabilizing controllers," Proceedings of 2006 American Control Conference, Minneapolis, MN, June 2006.

[14] S. Jiang and P. G. Voulgaris, "Switching among structured stabilizing controllers," Proceedings of the 46th IEEE Conference on Decision and Control, New Orleans, LA, December 2007.

[15] M. A. Dahleh and I. J. Diaz-Bobillo, Control of Uncertain Systems: A Linear Programming Approach, Prentice Hall, Englewood Cliffs, New Jersey, 1995.

[16] X. Qi, M. V. Salapaka, P. G. Voulgaris, and M. Khammash, "Structured optimal and robust control with multiple criteria: a convex solution," IEEE Transactions on Automatic Control, Vol. 49, No. 10, pp. 16231640, October 2004.

[17] M. Rotkowitz and S. Lall, "A characterization of convex problems in decentralized control," IEEE Transactions on Automatic Control, Vol. 51, No. 1, pp. 1984-1996, February 2006. 